UG-FT-282/11

CAFPE-152/11

\title{
Heavy-neutrino decays at neutrino telescopes
}

\author{
Manuel Masip, Pere Masjuan \\ CAFPE and Departamento de Física Teórica y del Cosmos \\ Universidad de Granada, E-18071, Granada, Spain \\ masip@ugr.es, masjuan@ugr.es
}

\begin{abstract}
It has been recently proposed that a sterile neutrino $\nu_{h}$ of mass $m_{h}=40-80 \mathrm{MeV}$, mixing $\left|U_{\mu h}\right|^{2} \approx 10^{-3}-10^{-2}$, lifetime $\tau_{h} \lesssim 10^{-9} \mathrm{~s}$, and a dominant decay mode $\nu_{h} \rightarrow \nu \gamma$ could be the origin of the experimental anomalies observed at LSND, KARMEN and MiniBooNE. Such a particle would be abundant inside air showers, as it can be produced in kaon decays $\left(K \rightarrow \nu_{h} \mu, K_{L} \rightarrow \nu_{h} \pi \mu\right)$. We use the $Z$-moment method to evaluate its atmospheric flux and the frequency of its decays inside neutrino telescopes. We show that $\nu_{h}$ would imply around $10^{4}$ contained showers per year inside a $0.03 \mathrm{~km}^{3}$ telescope like ANTARES or the DeepCore in IceCube. These events would have a characteristic energy and zenith-angle distribution $\left(E_{\nu} \approx 0.1-10 \mathrm{TeV}\right.$ and $\left.\theta<90^{\circ}\right)$, which results from a balance between the reach of the heavy neutrino (that disfavors low energies) and a sizeable production rate and decay probability. The standard background from contained neutrino events $\left(\nu_{e} N \rightarrow e X\right.$ and neutral-current interactions of high inelasticity) is 100 times smaller. Therefore, although it may be challenging from an experimental point of view, a search at ANTARES and IceCube could confirm this heavy-neutrino possibility.
\end{abstract}




\section{Introduction}

The direct observation of neutrino interactions in different types of experiments [1] has been used to establish a basic picture of neutrino masses and mixings. From a model building point of view, this is arguably the most significant discovery occurred in particle physics since the confirmation of the standard model in the early 70's, as it reveals a scale that is (most likely) not electroweak. The picture, however, has faced some persistent anomalies in experiments with neutrino beams from particle accelerators. Basically, muon neutrinos of energy below $1 \mathrm{GeV}$ seem to experience an excess of charged-current (CC) interactions with an electron in the final state. The interpretation of these events in terms of $\nu_{\mu} \rightarrow \nu_{e}$ oscillations is inconsistent with the mass parameters deduced from solar, atmospheric and reactor neutrino observations.

In a recent analysis Gninenko 2] has made a very compelling case for a massive neutrino as the origin for all these anomalies:

(i) LSND [3] observed $\bar{\nu}_{e}$-like events $\left(\bar{\nu}_{e} p \rightarrow e^{+} n\right)$ with a gamma signal from neutron capture that seem to imply an excess of $\bar{\nu}_{\mu} \rightarrow \bar{\nu}_{e}$ oscillations. He shows that the events could be equally explained through $\nu_{h}$ production $\left(\nu_{\mu}^{12} C \rightarrow \nu_{h} n X\right)$ followed by its radiative decay $\nu_{h} \rightarrow \nu \gamma$, with the final $\gamma$ converted into a $e^{+} e^{-}$pair indistinguishable from an electron. This explanation would work for a large enough production cross section $\left(\left|U_{\mu h}\right|^{2} \approx 10^{-3}-10^{-2}\right.$ and $\left.m_{\nu}<80 \mathrm{MeV}\right)$ and a short enough decay length $\left(\tau_{h} \lesssim 10^{-9} \mathrm{~s}\right.$ and $\left.m_{\nu}>40 \mathrm{MeV}\right)$. During the first years of data taking LSND also observed an excess of $\nu_{e}^{12} C \rightarrow e^{-} X$ events that were interpreted as $\nu_{\mu} \rightarrow \nu_{e}$ oscillations but are consistent as well with the $\nu_{h}$ hypothesis.

(ii) KARMEN [4], using a similar technique, did not confirm the LSND anomalies. The neutrinos at LSND, however, had an average energy of $100 \mathrm{MeV}$ and a long high-energy tail, whereas the spectrum at KARMEN was a narrow peak around $20 \mathrm{MeV}$. Gninenko shows that a $40 \mathrm{MeV}$ neutrino would be above the production threshold at KARMEN, which makes his hypothesis consistent with the data.

(iii) MiniBooNE [5] has observed an excess of electron-like events for $\nu_{e}$ energies between 200 and $475 \mathrm{MeV}$, with no significant excess at higher energies. Gninenko's fit exhibits also a good agreement with the data (higher-energy events are disfavored by an increase in the decay length and are hidden by the low statistics). More recently [6] this experiment has also reported an excess in $\bar{\nu}_{\mu}$ data for antineutrino energies in a wider range. His fit is consistent as well, and could favor a Dirac nature for $\nu_{h}$.

In addition, the mass range $40 \leq m_{h} \leq 80 \mathrm{MeV}$ makes $\nu_{h}$ too heavy to be produced in pion decays and too light to distort the muon spectrum in kaon decays. The heavy neutrino is also 
produced when the muon itself decays, but he argues that a mixing $\left|U_{\mu h}\right|^{2}<10^{-2}$ makes it acceptable. Specific searches for unstable neutrinos put strong constraints on $\left|U_{\mu h}\right|$, but are based on decays with charged particles in the final state $\left(\nu_{h} \rightarrow e e \nu, \mu e \nu, \mu \pi \nu\right)$, never on the decay $\nu_{h} \rightarrow \nu \gamma$ induced by a magnetic moment transition. Its large mass and short lifetime should keep $\nu_{h}$ also safe from bounds from supernovae and primordial nucleosynthesis [7]. Finally, a recent analysis [8] of muon capture with photon emission at TRIUMF finds that Gninenko's neutrino would imply a signal well above the $30 \%$ excess (versus the standard model value) deduced from the data [9]. One should notice, however, that the photon energy cut and the small size of the target volume at TRIUMF make this experiment very sensitive to the neutrino lifetime. A value $\tau_{h} \approx 3 \times 10^{-9} \mathrm{~s}$ could imply a consistent radiative capture rate there while explaining the data at LSND and KARMEN (which require $\tau_{h} \leq 10^{-8} \mathrm{~s}$ ) and still having an impact at MiniBooNE1.

We find the heavy neutrino hypothesis very interesting and will study here its implications in a different type of experiments. Our basic observation is that $\nu_{h}$ would be abundantly produced in the atmosphere through kaon decays. At energies around $1 \mathrm{TeV}$ its decay length becomes $c \tau_{h} \gamma \approx 5 \mathrm{~km}$, which implies that $\nu_{h}$ can reach a neutrino telescope and then decay. The final photon would be seen there as a pointlike event, similar to the shower from $\nu_{e} N \rightarrow e X$ or from a neutral-current (NC) interaction of high inelasticity but clearly distinguishable from the muon track in $\nu_{\mu} N \rightarrow \mu X$.

\section{$2 \quad$ Neutrino fluxes at sea level}

The atmospheric flux of any species can be easily estimated using the $Z$-moment method [10, 11]. This method provides a set of coupled differential equations that describe the evolution with the atmospheric depth $t$ (in $\left.\mathrm{g} / \mathrm{cm}^{2}\right)$ of the fluxes of parent hadrons $\left(\phi_{H}\right.$ with $\left.H=p, n, \pi^{ \pm}, K^{ \pm}, K_{L}\right)$ and of any particles that may result from their decay or their collision with an air nucleus. The generic equations for $\phi_{H}(E, \theta, t)$ are

$$
\frac{\partial \phi_{H}}{\partial t}=-\frac{\phi_{H}}{\lambda_{\mathrm{dec}}^{H}}-\frac{\phi_{H}}{\lambda_{\mathrm{int}}^{H}}+\sum_{H^{\prime}} S_{H^{\prime} H}
$$

where $\lambda_{\mathrm{dec}}^{H}\left(\lambda_{\mathrm{int}}^{H}\right)$ is the decay (interaction) length of $H$ in the air and $S_{H^{\prime} H}$ describe the sources. These equations can be solved analytically under some simplifying assumptions, namely,

(i) the all-nucleon primary flux has a constant spectral index $-\alpha$;

\footnotetext{
${ }^{1}$ A global fit including TRIUMF would certainly constrain further the parameter space in Gninenko's model.
} 
(ii) the energy distribution of particles from collisions and decays scales linearly with the energy of the parent hadron;

(iii) the hadronic interaction lengths $\lambda_{\text {int }}^{H}$ do not change with the energy;

(iv) the contributions to the nucleon flux from meson collisions and to the pion flux from kaon collisions are negligible.

It follows that the nucleon fluxes $\phi_{N}$ keep the same spectral index $-\alpha$ at any depth, and that the source terms are reduced to

$$
S_{N H}=\frac{\phi_{N}}{\lambda_{\text {int }}^{N}} Z_{N H}
$$

where the $Z$-factors

$$
Z_{N H}=\int_{0}^{1} \mathrm{~d} x x^{\alpha-1} F_{N H}
$$

are constants (independent of $E$ and the zenith angle $\theta$ ) derived from the distribution $F_{N H}(x)$ of the fraction of energy taken by $H$ after a $N$-air collision. The meson fluxes $\phi_{M}$ can then be easily derived in two different regimes. At low energies $\lambda_{\mathrm{dec}}^{M}=\left(E / m_{M}\right) c \tau_{M} \rho$ is much smaller than $\lambda_{\text {int }}^{M}$ and meson interactions can be ignored, whereas at high energies the variations in $\phi_{M}$ are dominated by collisions with air nuclei (the air density $\rho$ is a function of $t$ and of $\theta$ ). A simple interpolation can be used between these regimes. We take in our analysis the primary flux, the $Z$-factors and the atmospheric model in [11] (see [12] for a discussion of the fluxes at higher energies). We obtain, for example, that the $\mathrm{TeV}$ charged-pion vertical flux reaches its maximum (a $4 \%$ of the initial nucleon flux) at a depth of $200 \mathrm{~g} / \mathrm{cm}^{2}$, and that the kaon flux there is 7 times smaller.

The lepton fluxes from meson decays can also be incorporated. In particular, standard neutrinos do not interact nor decay in the atmosphere and their flux equations will only depend on source terms of type

$$
S_{M \nu}(E)=B(M \rightarrow \nu) \int_{0}^{1} \mathrm{~d} x x^{-1} \frac{\phi_{M}(E / x)}{\lambda_{\mathrm{dec}}^{M}(E / x)} F_{M \nu}(x),
$$

where $B(M \rightarrow \nu)$ is the branching ratio of a given decay mode, $F_{M \nu}(x)$ is again the distribution of the fraction of energy taken by the neutrino in that decay, and the dependence on $t$ and $\theta$ is implicit. We obtain that, although kaons are less abundant than pions in air showers, a lower ratio $\lambda_{\text {dec }}^{M} / \lambda_{\text {int }}^{M}$ makes them the main source of neutrinos at energies above $100 \mathrm{GeV}$. The TeV flux at sea level is dominated by muon neutrinos, with $\phi_{\bar{\nu}_{\mu}} \approx 0.42 \phi_{\nu_{\mu}}$, $\phi_{\nu_{e}} \approx 0.036 \phi_{\nu_{\mu}}$ and $\phi_{\bar{\nu}_{e}} \approx 0.023 \phi_{\nu_{\mu}}$.

Heavy neutrinos $\nu_{h}$ will be mainly produced in charged-kaon decays. The branching ratio is

$$
B\left(K^{+} \rightarrow \mu^{+} \nu_{h}\right) \approx B\left(K^{+} \rightarrow \mu^{+} \nu\right) \times\left|U_{\mu h}\right|^{2} \bar{\rho}_{h}
$$




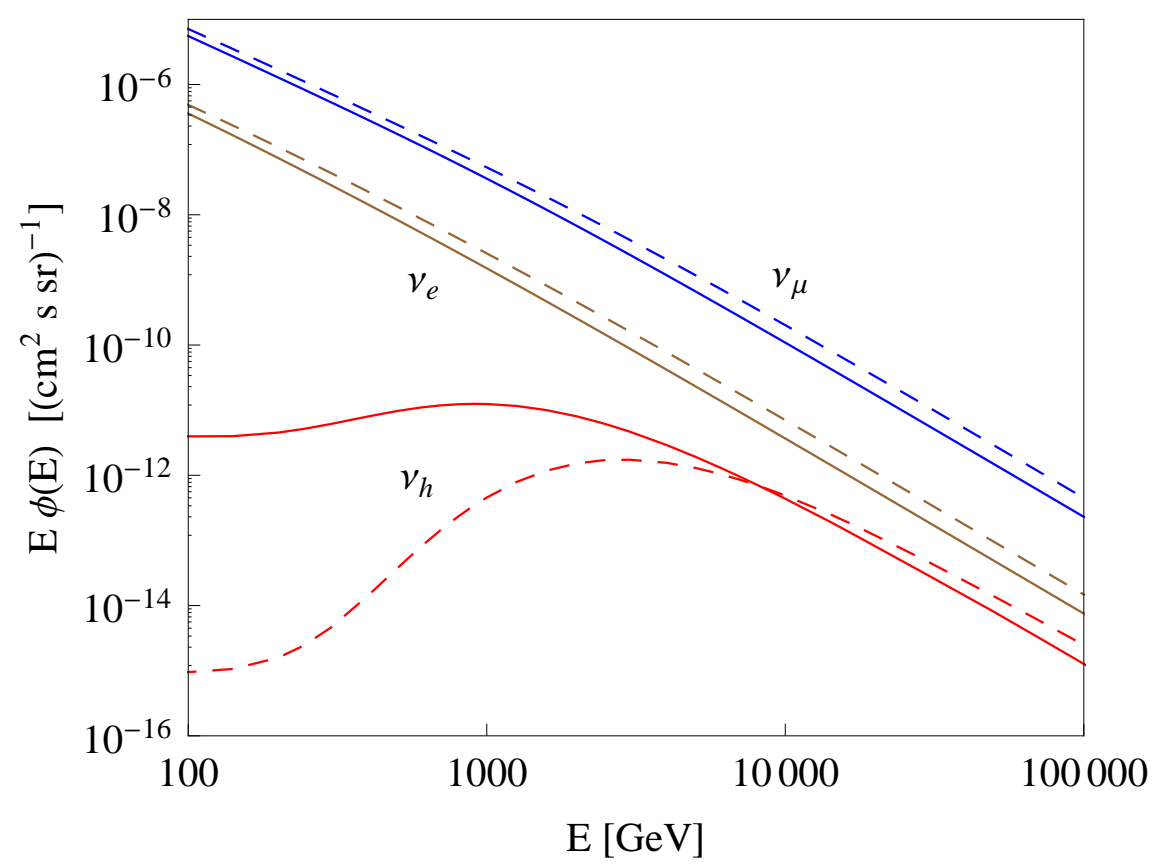

Figure 1: Neutrino fluxes $\left(\nu_{i}+\bar{\nu}_{i}\right)$ at sea level for $\theta=0$ (solid) and $\theta=60^{\circ}$ (dashes).

where $B\left(K^{+} \rightarrow \mu^{+} \nu\right)=0.64$ and the kinematic factor for $m_{h}=40-80 \mathrm{MeV}$ is $\bar{\rho}_{h} \approx$ $\left(1+m_{h}^{2} / m_{\mu}^{2}\right)$ [13]. The fraction of energy $x$ taken by $\nu_{h}$ will have a flat distribution (a constant $\left.F_{K h}(x)\right)$ between $x_{\min }$ and $x_{\max }$,

$$
x_{\substack{\min \\ \max }}=\frac{1}{2}\left(1+\frac{m_{h}^{2}-m_{\mu}^{2}}{m_{K}^{2}}\right) \mp \sqrt{\frac{1}{4}\left(1+\frac{m_{h}^{2}-m_{\mu}^{2}}{m_{K}^{2}}\right)-\frac{m_{h}^{2}}{m_{K}^{2}}} .
$$

There will be smaller contributions from $K^{+} \rightarrow \pi^{0} \mu^{+} \nu_{h}$ and $K_{L} \rightarrow \pi^{-} \mu^{+} \nu_{h}$, plus the analogous $K^{-}$and $K_{L}$ decays into $\bar{\nu}_{h}$ (the heavy neutrino may be a Dirac or a Majorana particle, see discussion in [2]). The equation defining $\phi_{h}(E, \theta, t)$ is

$$
\frac{\partial \phi_{h}}{\partial t}=-\frac{\phi_{h}}{\lambda_{\mathrm{dec}}^{h}}+\sum_{K} S_{K h}
$$

where the source terms take the form in Eq. (4),$\lambda_{\text {dec }}^{h}=\left(E / m_{h}\right) c \tau_{h} \rho$, and the sum runs over the decay modes that produce $\nu_{h}$. In Fig. 1 we plot the total heavy neutrino flux at sea level from inclinations $\theta=0^{\circ}, 60^{\circ}$. We have taken the central values $m_{h}=60 \mathrm{MeV}$ and $\left|U_{\mu h}\right|^{2}=0.005$, with $\tau_{h}=10^{-9} \mathrm{~s}$. At $1 \mathrm{TeV} 73 \%$ of the flux comes from $K^{+}$decays, $K^{-}$ contributes a $25 \%$, and $K_{L}$ just a $2 \%$. Finally, notice that the photons produced in the air through $\nu_{h}$ decays are together with other photons and muons inside the parent shower and are therefore non-observable. 


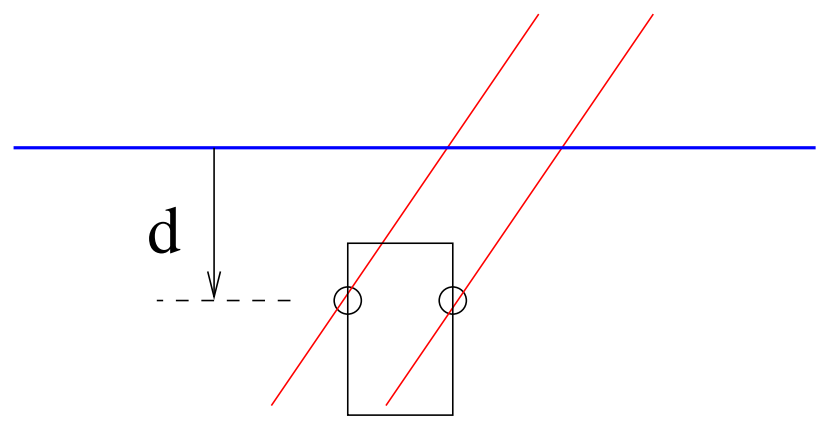

Figure 2: The total flux through the lateral surface of the detection region cancels if $R_{T} \gg$ $d / \cos \theta$ (we take $\theta \leq 85^{\circ}$ ).

\section{Events at a neutrino telescope}

As neutrinos enter the ground their sources disappear and they just experience two types of processes: heavy neutrinos $\nu_{h}$ may decay into $\gamma \nu_{\mu}$, whereas $\nu_{\mu}$ and $\nu_{e}$ may have neutralor charged-current interactions with matter. At a depth $d$ the sea-level fluxes $\phi_{i}(E, \theta, 0)$ become

$$
\begin{aligned}
& \phi_{h}(E, \theta, d)=\phi_{h}(E, \theta, 0) \exp \left(\frac{-d}{\lambda_{\mathrm{dec}}^{h} \cos \theta}\right) ; \\
& \phi_{\nu}(E, \theta, d)=\phi_{\nu}(E, \theta, 0) \exp \left(\frac{-d}{\lambda_{\text {int }}^{\nu} \cos \theta}\right),
\end{aligned}
$$

where $d$ and the decay/interaction lengths are given in meters and we have neglected the curvature of the Earth (a good approximation for $\theta \leq 85^{\circ}$ ). At $1 \mathrm{TeV}$ we obtain $\lambda_{\mathrm{dec}}^{h} \approx 5$ $\mathrm{km}$ whereas $\lambda_{\mathrm{CC}}^{\nu} \approx 2 \times 10^{6} \mathrm{~km}$ and $\lambda_{\mathrm{NC}}^{\nu} \approx 8 \times 10^{6} \mathrm{~km}$ (the interaction lengths decrease with the energy as $\left.1 / \sigma_{\nu N}\right)$. This means that the decay of a $\nu_{h}$ crossing a neutrino telescope is $10^{6}$ times more probable than the interaction of a standard neutrino. In addition, CC $\nu_{\mu}$ interactions will be clearly different from $\nu_{h}$ decays, as the final muon will produce a track hundreds of meters long [15]. The electromagnetic shower from a $\nu_{h}$ decay will be pointlike (it develops in a few meters), similar to the one produced by a $\mathrm{NC}$ interaction or a $\nu_{e} \mathrm{CC}$ process. These standard events, however, are suppressed by the lower $\nu_{e}$ fluxes $\left(\nu_{\mu} \rightarrow \nu_{e}\right.$ oscillations at $L \leq 100 \mathrm{~km}$ and $E \geq 100 \mathrm{GeV}$ are negligible) and the inelasticity distribution $(\propto 1 / y[14])$ in $\nu-N$ collisions.

Let us be more specific. To estimate the number of events per unit time occurred inside a telescope one needs to calculate the total flux (ingoing plus outgoing neutrinos) through the surface containing the detectors. We model this region as a cylinder of section $A$ and length $H$ starting at a depth $d_{0}$ (i.e., $d$ goes from $d_{0}$ to $d_{0}+H$ ). For a fixed angle $\theta \lesssim 85^{\circ}$ 


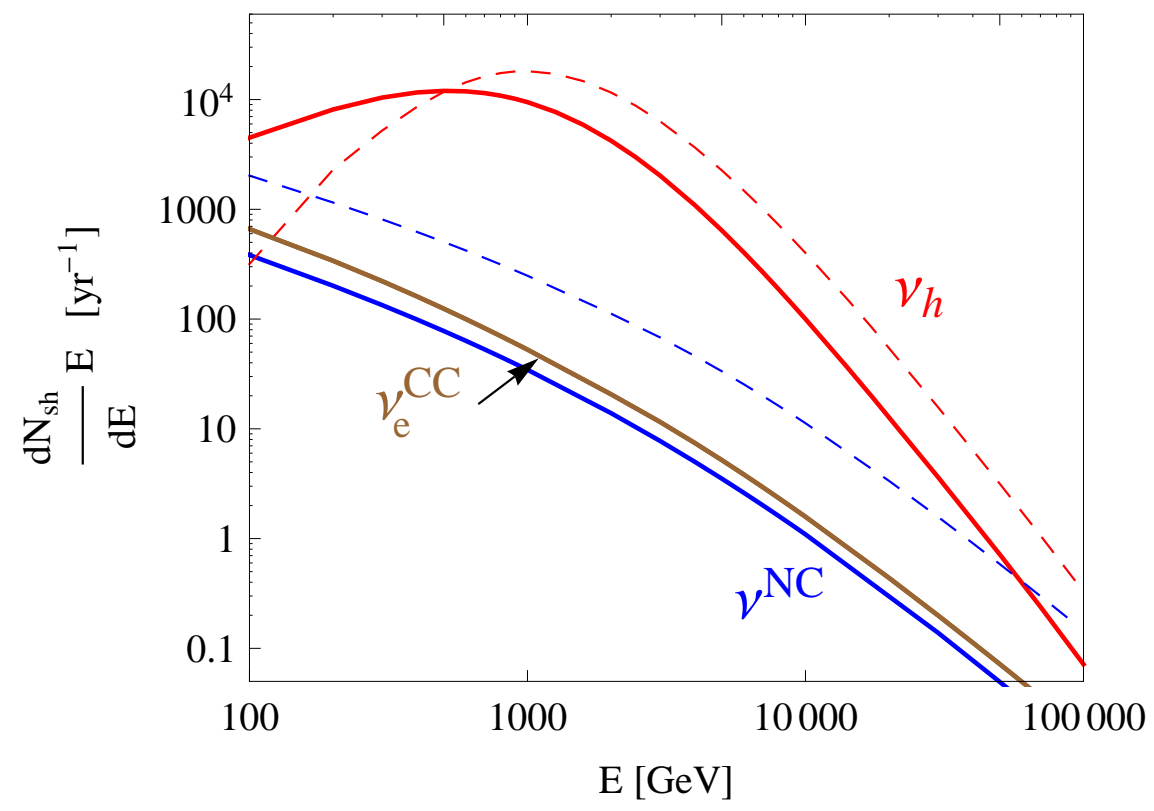

Figure 3: Energy distribution of the contained showers from $\nu_{h} \rightarrow \nu_{\mu} \gamma, \nu N \rightarrow \nu X$ and $\nu_{e} N \rightarrow e X$ at ANTARES. We also plot (dashes) the energy distribution of the parent neutrino in each case.

the neutrino flux only depends on the depth $d$. Therefore, the total flux through the lateral surface of the detector will be zero (given $\theta$, the flux through any lateral $\mathrm{d} \vec{S}$ is equal to the flux leaving the detector through an opposed lateral surface $-\mathrm{d} \vec{S}$, see Fig. 2). The number of heavy-neutrino events inside the detector in an interval of energy and solid angle per unit time can then be calculated as the difference between the fluxes through its upper and its lower sections:

$$
\begin{aligned}
N_{h}=\int_{\Delta E} \mathrm{~d} E \int_{\Delta \Omega} \mathrm{d} \Omega \int_{A} \mathrm{~d} S & \cos \theta\left[\phi_{h}\left(E, \theta, d_{0}\right)\right. \\
- & \left.\phi_{h}\left(E, \theta, d_{0}+H\right)\right] .
\end{aligned}
$$

An analogous expression can be obtained to estimate the number $N_{\nu}$ of interactions inside the detector produced by neutrinos from those directions. In Fig. 3 we plot in dashes the energy distribution of the neutrinos that interact $\left(\nu_{\mu}\right.$ and $\left.\nu_{e}\right)$ or decay $\left(\nu_{h}\right)$ per year inside a detector like ANTARES [16] $\left(A=0.1 \mathrm{~km}^{2}, d_{0}=2.2 \mathrm{~km}, H=0.3 \mathrm{~km}\right)$ or the DeepCore [17] in IceCube (of similar size and depth). The energy of the initial neutrino, however, is not the most relevant parameter for observation, as in NC interactions only a small fraction $y$ may be deposited in the detector. In a $\nu_{h}$ decay only the photon energy is visible (we will assume an isotropic decay [2]), whereas in $\nu_{e}$-CC interactions all the energy carried by the 
neutrino goes to the contained shower. If the inelasticity $y$ in the event has a distribution $F_{\nu \mathrm{sh}}(y)$, then the energy distribution of the cascades inside the detector will be

$$
\frac{\mathrm{d} N_{\mathrm{sh}}}{\mathrm{d} E}(E)=\int_{0}^{1} \mathrm{~d} y y^{-1} \frac{\mathrm{d} N_{\nu}}{\mathrm{d} E}(E / y) F_{\nu \mathrm{sh}}(y) .
$$

Fig. 3 shows in solid lines the main result of our analysis. The number of standard showers of energy above $100 \mathrm{GeV}$ from down-going neutrinos inside ANTARES is around 1300 per year (60\% from $\nu_{e}$-CC interactions and $40 \%$ from $\mathrm{NC}$ interactions). For the central values $m_{h}=60 \mathrm{MeV}$ and $\left|U_{\mu h}\right|^{2}=0.005$ the heavy neutrino would provide 26000 extra events. If the energy threshold is set at $500 \mathrm{GeV}$ the number of standard events is reduced to 220 per year, whereas the number of events from $\nu_{h}$ decays is just cut to 14000 .

\section{Summary and discussion}

Telescopes like ANTARES or IceCube are designed to observe upward-moving muons produced in neutrino interactions near the detector. These events are clean, in the sense that no particles except for neutrinos can reach the detector after crossing the Earth. Telescopes can also observe the contained showers produced in $\mathrm{NC}$ interactions or in $\nu_{e}$-CC processes. Since their development takes just a few meters, these events are pointlike, and the only sign indicating whether they are caused by an upward or a downward-going neutrino is that in the latter case they may come together with muons.

In this paper we have shown that the decay of a long-lived neutral particle produced in the atmosphere could change drastically (by over a factor of 100) the number of $\mathrm{TeV}$ contained showers in these experiments. In particular, we have analyzed Gninenko's heavy neutrino, that appears as a possibility well motivated by the results at LSND, KARMEN and MiniBooNE. We find remarkable that its mass, mixing, and lifetime optimize the distortion introduced in $\mathrm{TeV}$-neutrino telescopes: below $\approx 100 \mathrm{GeV} \nu_{h}$ does not reach the telescope, and above $100 \mathrm{TeV}$ its decay length becomes too large and the signal vanishes (notice that $\lambda_{\mathrm{dec}}^{h}$ grows with the energy while $\lambda_{\text {int }}^{\nu}$ decreases).

The heavy neutrino would be produced through kaon decays inside air showers together with a muon of similar energy. A crucial question is then whether these decays can be disentangled from the muon bundle associated to the parent shower. ANTARES or the DeepCore in IceCube are more than $2 \mathrm{~km}$ deep, and as the zenith angle grows all muon effects will decrease. In contrast, the zenith angle dependence of the $\nu_{h}$ events up to $85^{\circ}$ is very mild (especially at larger energies), since these neutrinos do not lose energy in their way to the detector. Therefore, the $\mathrm{TeV}$ contained showers should appear as a clear anomaly that 
may be accompanied by muons in vertical events but that persists at higher zenith angles (slant depths). The large number of events that we obtain could allow for specific searches. For example, events with lower-energy muons in the upper part of the detector followed by a contained $\mathrm{TeV}$ shower below, or other topologies that would otherwise be discarded.

If a heavy neutrino is the explanation of the LSND and MiniBooNE anomalies, our results show that it will reach effectively the core of neutrino telescopes and will decay there at a high rate. A MonteCarlo simulation of individual showers, including all the muon backgrounds and the response of the detector, should then provide the best strategy in the search for an observable signal that confirms or excludes this heavy neutrino possibility.

\section{Acknowledgments}

We would like to thank Juande Zornoza for discussions. This work has been partially supported by MICINN of Spain (FPA2006-05294, FPA2010-16802, FPA2010-16696, ConsoliderIngenio Multidark CSD2009-00064 and CPAN CSD2007-00042) and by Junta de Andalucía (FQM 101, FQM 437 and FQM 3048).

\section{References}

[1] J. M. Conrad, "Neutrino Experiments," arXiv:0708.2446 [hep-ex].

[2] S. N. Gninenko, Phys. Rev. D 83 (2011) 015015.

[3] C. Athanassopoulos et al. [LSND Collaboration], Phys. Rev. Lett. 77 (1996) 3082;

C. Athanassopoulos et al. [LSND Collaboration], Phys. Rev. C 54 (1996) 2685;

C. Athanassopoulos et al. [LSND Collaboration], Phys. Rev. Lett. 81 (1998) 1774;

A. Aguilar et al. [LSND Collaboration], Phys. Rev. D 64 (2001) 112007.

[4] B. Armbruster et al. [KARMEN Collaboration], Phys. Rev. D 65 (2002) 112001.

[5] A. A. Aguilar-Arevalo et al. [The MiniBooNE Collaboration], Phys. Rev. Lett. 98 (2007) 231801; A. A. Aguilar-Arevalo et al. [MiniBooNE Collaboration], Phys. Rev. Lett. 102 (2009) 101802.

[6] A. A. Aguilar-Arevalo et al. [The MiniBooNE Collaboration], Phys. Rev. Lett. 105 (2010) 181801. 
[7] A. D. Dolgov, S. H. Hansen, G. Raffelt and D. V. Semikoz, Nucl. Phys. B 580 (2000) 331; Nucl. Phys. B 590 (2000) 562.

[8] D. McKeen and M. Pospelov, Phys. Rev. D 82 (2010) 113018.

[9] V. Bernard, T. R. Hemmert and U. G. Meissner, Nucl. Phys. A 686 (2001) 290 arXiv:nucl-th/0001052.

[10] T.K. Gaisser, "Cosmic Rays and Particle Physics", Cambridge University Press (1990).

[11] P. Lipari, Astropart. Phys. 1 (1993) 195.

[12] J. I. Illana, P. Lipari, M. Masip and D. Meloni, Astropart. Phys. 34 (2011) 663.

[13] R. E. Shrock, Phys. Rev. D 24 (1981) 1232.

[14] A. Connolly, R. S. Thorne and D. Waters, "Calculation of High Energy NeutrinoNucleon Cross Sections and Uncertainties Using the MSTW Parton Distribution Functions and Implications for Future Experiments," arXiv:1102.0691 [hep-ph].

[15] F. Halzen and S. R. Klein, Rev. Sci. Instrum. 81 (2010) 081101.

[16] J. Brunner [ANTARES Collaboration], Nucl. Instrum. Meth. A 626-627 (2011) S19.

[17] C. Wiebusch and f. t. I. Collaboration, arXiv:0907.2263 [astro-ph.IM]. 